\title{
GROUND STATES OF NONLOCAL SCALAR FIELD EQUATIONS WITH TRUDINGER-MOSER CRITICAL NONLINEARITY
}

\author{
JOÃO MARCOS DO Ó, OLÍMPIO H. MIYAGAKI, AND MARCO SQUASSINA
}

\begin{abstract}
We investigate the existence of ground state solutions for a class of nonlinear scalar field equations defined on whole real line, involving a fractional Laplacian and nonlinearities with Trudinger-Moser critical growth. We handle the lack of compactness of the associated energy functional due to the unboundedness of the domain and the presence of a limiting case embedding.
\end{abstract}

\section{INTRODUCTION AND MAIN RESUlT}

The goal of this paper is to investigate the existence of ground state solutions $u \in H^{1 / 2}(\mathbb{R})$ for the following class of nonlinear scalar field equations

$$
(-\Delta)^{1 / 2} u+u=f(u) \quad \text { in } \mathbb{R},
$$

where $f: \mathbb{R} \rightarrow \mathbb{R}$ is a smooth nonlinearity in the critical growth range. Precisely, we focus here on the case when $f$ has the maximal growth which allows to study problem (1.1) variationally in the Sobolev space $u \in H^{1 / 2}(\mathbb{R})$, see Section 2. We are motivated by the following Trudinger-Moser type inequality due to Ozawa [28].

Theorem A. There exists $0<\omega \leq \pi$ such that, for all $\alpha \in(0, \omega)$, there exists $H_{\alpha}>0$ with

$$
\int_{\mathbb{R}}\left(e^{\alpha u^{2}}-1\right) \mathrm{d} x \leq H_{\alpha}\|u\|_{L^{2}}^{2},
$$

for all $u \in H^{1 / 2}(\mathbb{R})$ with $\left\|(-\Delta)^{1 / 4} u\right\|_{L^{2}}^{2} \leq 1$.

From inequality (1.2) we have naturally associated notions of subcriticality and criticality for this class of problems. Precisely, we say that $f: \mathbb{R} \rightarrow \mathbb{R}$ has subcritical growth at $\pm \infty$ if

$$
\limsup _{s \rightarrow \pm \infty} \frac{f(s)}{e^{\alpha s^{2}}-1}=0, \quad \text { for all } \alpha>0,
$$

and has $\alpha_{0}$-critical growth at $\pm \infty$ if there exists $\omega \in(0, \pi]$ and $\alpha_{0} \in(0, \omega)$ such that

$$
\begin{aligned}
& \limsup _{s \rightarrow \pm \infty} \frac{f(s)}{e^{\alpha s^{2}}-1}=0, \quad \text { for all } \alpha>\alpha_{0}, \\
& \limsup _{s \rightarrow \pm \infty} \frac{f(s)}{e^{\alpha s^{2}}-1}= \pm \infty, \quad \text { for all } \alpha<\alpha_{0} .
\end{aligned}
$$

For instance let $f$ be given by

$$
f(s)=s^{3} e^{\alpha_{0}|s|^{\nu}} \quad \text { for all } s \in \mathbb{R} .
$$

2000 Mathematics Subject Classification. 35J60, 35B09, 35B33, 35R11.

Key words and phrases. Trudinger-Moser inequality, fractional Laplacian, ground state solutions.

The present research was partially supported by INCTmat/MCT/Brazil. João Marcos do Ó was supported by CNPq, CAPES/Brazil, Olímpio H. Miyagaki was partially supported by CNPq/Brazil and CAPES/Brazil (Proc 2531/14-3). The paper was completed while the second author was visiting the Dept of Math of Rutgers University, whose hospitality he gratefully acknowledges. 
If $\nu<2, f$ has subcritical growth, and while if $\nu=2$ and $\alpha_{0} \in(0, \omega], f$ has critical growth. By a ground state solution to problem (1.1) we mean a nontrivial weak solution of (1.1) with the least possible energy.

The following assumptions on $f$ will be needed throughout the paper:

(f1): $f: \mathbb{R} \rightarrow \mathbb{R}$ is $C^{1}$, odd, convex function on $\mathbb{R}^{+}$, and

$$
\lim _{s \rightarrow 0} \frac{f(s)}{s}=0 .
$$

(f2): $s \mapsto s^{-1} f(s)$ is an increasing function for $s>0$.

(f3): there are $q>2$ and $C_{q}>0$ with

$$
F(s) \geq C_{q}|s|^{q}, \quad \text { for all } s \in \mathbb{R} .
$$

(AR): there exists $\vartheta>2$ such that

$$
\vartheta F(s) \leq s f(s), \quad \text { for all } s \in \mathbb{R}, \quad F(s)=\int_{0}^{s} f(\sigma) \mathrm{d} \sigma .
$$

The main result of the paper is the following

Theorem 1.1. Let $f(s)$ and $f^{\prime}(s)$ s have $\alpha_{0}$-critical growth and satisfy $(\mathbf{f 1})-(\mathbf{f 3})$ and $(\mathbf{A R})$. Then problem (1.1) admits a ground state solution $u \in H^{1 / 2}(\mathbb{R})$ provided $C_{q}$ in (f3) is large enough.

The nonlinearity

$$
f(s)=\lambda s|s|^{q-2}+|s|^{q-2} s e^{\alpha_{0} s^{2}}, \quad q>2 \text { and } s \in \mathbb{R},
$$

satisfies all the hypotheses of Theorem 1.1 provided that $\lambda$ is sufficiently large. More examples of nonlinearities which satisfy the above assumptions can be found in [19]. In $\mathbb{R}^{2}$ one can use radial estimates, then apply, for instance, the Strauss lemma [32] to recover some compactness results. In $\mathbb{R}$ analogous compactness results fail, but in [21], the authors used the concentration compactness principle by Lions [34] for problems with polynomial nonlinearities. In this paper, we use the minimization technique over the Nehari manifold in order to get ground state solutions. We adopt some arguments from [4] combined with those used in [10,23].

1.0.1. Quick overview of the literature. In Coti Zelati and Rabinowitz [12] investigated

$$
-\Delta u+V(x) u=f(x, u) \quad \text { in } \mathbb{R}^{N}, \quad u \in H^{1}\left(\mathbb{R}^{N}\right), \quad u>0,
$$

when $V$ is a strictly positive potential and $f: \mathbb{R}^{N} \times \mathbb{R} \rightarrow \mathbb{R}$ is a periodic function in $x \in \mathbb{R}^{N}$ and $f$ has Sobolev subcritical growth, that is, $f$ behaves at infinity like $s^{p}$ with $2<p<2^{*}-1$, where $2^{*}=2 N /(N-2)$ is the critical Sobolev exponent, $N \geq 3$. This was extended or complemented in several ways, see e.g. [34]. For $N=2$ formally $2^{*} \leadsto+\infty$, but $H^{1}\left(\mathbb{R}^{N}\right) \hookrightarrow L^{\infty}\left(\mathbb{R}^{N}\right)$. Instead, the Trudinger-Moser inequality $[27,33]$ states that $H^{1}$ is continuously embedded into an Orlicz space defined by the Young function $\phi(t)=e^{\alpha t^{2}}-1$. In $[1,14,15,25]$, with the help of TrudingerMoser embedding, problems in a bounded domain were investigated, when the nonlinear term $f$ behaves at infinity like $e^{\alpha s^{2}}$ for some $\alpha>0$. We refer the reader to [13] for a recently survey on this subject. In [11] the Trudinger-Moser inequality was extended to the whole $\mathbb{R}^{2}$ and the authors gave some applications to study equations like (1.3) when the nonlinear term has critical growth of Trudinger-Moser type. For further results and applications, we would like to mention also $[2,3,17,29]$ and references therein. When the potential $V$ is a positive constant and $f(x, s)=f(s)$ for $(x, s) \in \mathbb{R}^{N} \times \mathbb{R}$, that is the autonomous case, the existence of ground states for subcritical nonlinearities was established in [6] for $N \geq 3$ and [7] for $N=2$ respectively, while in [3] the critical case for $N \geq 3$ and $N=2$ was treated. For fractional problem of the form

$$
(-\Delta)^{s} u+V(x) u=f(u) \quad \text { in } \mathbb{R}^{N},
$$


with $N>2 s$ and $s \in(0,1)$, we refer to $[10,20]$ where positive ground states were obtained in subcritical situations. For instance, [10] extends the results in [6] to the fractional Laplacian. In [20] is obtained regularity and qualitative properties of the ground state solution, while in [31] a ground state solution is obtained for coercive potential. For fractional problems in bounded domains of $\mathbb{R}^{N}$ with $N>2 s$ involving critical nonlinearities we cite $[5,9,22,30]$ and [18] for the whole space with vanishing potentials. In [21] the authors investigated properties of the ground state solutions of $(-\Delta)^{s} u+u=u^{p}$ in $\mathbb{R}$. Recently, in [23], nonlocal problems defined in bounded intervals of the real line involving the square root of the Laplacian and exponential nonlinearities were investigated, using a version of the Trudinger-Moser inequality due to Ozawa [28]. As it was remarked in [23] the nonlinear problem involving exponential growth with fractional diffusion $(-\Delta)^{s}$ requires $s=1 / 2$ and $N=1$. In [19] some nonlocal problems in $\mathbb{R}$ with vanishing potential, thus providing compactifying effects, are considered.

\section{Preliminary StUfF}

We recall that

$$
H^{1 / 2}(\mathbb{R})=\left\{u \in L^{2}(\mathbb{R}): \int_{\mathbb{R}^{2}} \frac{(u(x)-u(y))^{2}}{|x-y|^{2}} \mathrm{~d} x \mathrm{~d} y<\infty\right\},
$$

endowed with the norm

$$
\|u\|=\left(\|u\|_{L^{2}}^{2}+\int_{\mathbb{R}^{2}} \frac{(u(x)-u(y))^{2}}{|x-y|^{2}} \mathrm{~d} x \mathrm{~d} y\right)^{1 / 2} .
$$

The square root of the Laplacian, $(-\Delta)^{1 / 2}$, of a smooth function $u: \mathbb{R} \rightarrow \mathbb{R}$ is defined by

$$
\mathcal{F}\left((-\Delta)^{1 / 2} u\right)(\xi)=|\xi| \mathcal{F}(u)(\xi),
$$

where $\mathcal{F}$ denotes the Fourier transform, that is,

$$
\mathcal{F}(\phi)(\xi)=\frac{1}{\sqrt{2 \pi}} \int_{\mathbb{R}} e^{-i \xi \cdot x} \phi(x) \mathrm{d} x,
$$

for functions $\phi$ in the Schwartz class. Also $(-\Delta)^{1 / 2} u$ can be equivalently represented [16] as

$$
(-\Delta)^{1 / 2} u=-\frac{1}{2 \pi} \int_{\mathbb{R}} \frac{u(x+y)+u(x-y)-2 u(x)}{|y|^{2}} \mathrm{~d} y .
$$

Also, in light of [16, Propostion 3.6], we have

$$
\left\|(-\Delta)^{1 / 4} u\right\|_{L^{2}}^{2}:=\frac{1}{2 \pi} \int_{\mathbb{R}^{2}} \frac{(u(x)-u(y))^{2}}{|x-y|^{2}} \mathrm{~d} x \mathrm{~d} y, \quad \text { for all } u \in H^{1 / 2}(\mathbb{R}),
$$

and, sometimes, we identify these two quantities by omitting the normalization constant $1 / 2 \pi$. From [26, (iii) of Theorem 8.5] we also know that, for any $m \geq 2$, there exists $C_{m}>0$ such that

$$
\|u\|_{L^{m}} \leq C_{m}\|u\|, \quad \text { for all } u \in H^{1 / 2}(\mathbb{R}) .
$$

Proposition 2.1. The integral

$$
\int_{\mathbb{R}}\left(e^{\alpha u^{2}}-1\right) \mathrm{d} x
$$

is finite for any positive $\alpha$ and $u \in H^{1 / 2}(\mathbb{R})$. 
Proof. Let $\alpha_{0} \in(0, \omega)$ and consider the convex function defined by

$$
\phi(t)=\frac{e^{\alpha_{0} t^{2}}-1}{H_{\alpha_{0}}}, \quad t \in \mathbb{R},
$$

where $H_{\alpha_{0}}>0$ is defined as in Theorem A. We introduce the Orlicz norm induced by $\phi$ by setting

$$
\|u\|_{\phi}:=\inf \left\{\gamma>0: \int_{\mathbb{R}} \phi\left(\frac{u}{\gamma}\right) \mathrm{d} x \leq 1\right\}
$$

and the corresponding Orlicz space $L_{\phi^{*}}(0,1)$, see the monograph by Krasnosel'skiü \& Rutickiü [24, Chapter II, in particular p.78-81] for properties of this space. We claim that $\|v\|_{\phi} \leq\|v\|$, for all $v \in H^{1 / 2}(\mathbb{R})$. Let $v \in H^{1 / 2}(\mathbb{R}) \backslash\{0\}$ and set $w=\|v\|^{-1} v$, so that by formula (2.1) we conclude

$$
\left\|(-\Delta)^{\frac{1}{4}} w\right\|_{L^{2}}=\frac{1}{(2 \pi)^{\frac{1}{2}}\|v\|}\left(\int_{\mathbb{R}^{2}} \frac{(v(x)-v(y))^{2}}{|x-y|^{2}} \mathrm{~d} x \mathrm{~d} y\right)^{1 / 2} \leq(2 \pi)^{-\frac{1}{2}}<1 .
$$

Therefore, in light of Theorem A, we have

$$
\int_{\mathbb{R}} \phi\left(\frac{v}{\|v\|}\right) \mathrm{d} x=\int_{\mathbb{R}} \frac{e^{\alpha_{0} w^{2}}-1}{H_{\alpha_{0}}} \mathrm{~d} x \leq\|w\|_{L^{2}}^{2} \leq 1,
$$

which proves the claim by the very definition of $\|\cdot\|_{\phi}$. Fix now an arbitrary function $u \in H^{1 / 2}(\mathbb{R})$. Hence, there exists a sequence $\left(\psi_{n}\right)$ in $C_{c}^{\infty}(\mathbb{R})$ such that $\psi_{n} \rightarrow u$ in $H^{1 / 2}(\mathbb{R})$, as $n \rightarrow \infty$. By the claim this yields $\left\|\psi_{n}-u\right\|_{\phi} \rightarrow 0$, as $n \rightarrow \infty$. Fix now $n=n_{0}$ sufficiently large that $\left\|\psi_{n_{0}}-u\right\|_{\phi}<1 / 2$. Then we have, in light of [24, Theorem 9.15, p.79], that

$$
\int_{\mathbb{R}} \phi\left(2 u-2 \psi_{n_{0}}\right) \mathrm{d} x \leq\left\|2 u-2 \psi_{n_{0}}\right\|_{\phi}<1 .
$$

Finally, writing $u=\frac{1}{2}\left(2 u-2 \psi_{n_{0}}\right)+\frac{1}{2}\left(2 \psi_{n_{0}}\right)$, and since

$$
\int_{\mathbb{R}} \phi\left(2 \psi_{n_{0}}\right) \mathrm{d} x=\frac{1}{H_{\alpha_{0}}} \int_{\mathbb{R}}\left(e^{4 \alpha_{0} \psi_{n_{0}}^{2}}-1\right) \mathrm{d} x=\frac{1}{H_{\alpha_{0}}} \int_{\operatorname{supt}\left(\psi_{n_{0}}\right)}\left(e^{4 \alpha_{0} \psi_{n_{0}}^{2}}-1\right) \mathrm{d} x<\infty,
$$

the convexity of $\phi$ yields $\int_{\mathbb{R}} \phi(u) \mathrm{d} x<\infty$. Hence, the assertion follows by the arbitrariness of $u$. A different proof can be given writing (in the above notations)

$$
\int_{\mathbb{R}}\left(e^{\alpha u^{2}}-1\right) \mathrm{d} x=\int_{\mathbb{R}}\left(e^{\alpha \psi_{n}^{2}}-1\right) \mathrm{d} x+\int_{\mathbb{R}}\left(e^{\alpha u^{2}}-e^{\alpha \psi_{n}^{2}}\right) \mathrm{d} x,
$$

estimating the right-hand side by $\left|e^{\alpha u^{2}}-e^{\alpha \psi_{n}^{2}}\right| \leq 2 \alpha\left(\left|\psi_{n}-u\right|+\left|\psi_{n}\right|\right) e^{2 \alpha\left|\psi_{n}-u\right|^{2}} e^{2 \alpha\left|\psi_{n}\right|^{2}}\left|\psi_{n}-u\right|$, using Hölder inequality, the smallness of $\left\|\psi_{n}-u\right\|$ and Theorem A to conclude, for $n$ large enough.

Define the functional $J: H^{1 / 2}(\mathbb{R}) \rightarrow \mathbb{R}$ associated with problem (1.1), given by

$$
J(u)=\frac{1}{2} \int_{\mathbb{R}}\left(\left|(-\Delta)^{1 / 4} u\right|^{2}+u^{2}\right) \mathrm{d} x-\int_{\mathbb{R}} F(u) \mathrm{d} x .
$$

Under our assumptions on $f$, by Proposition 2.1 we can easily see that $J$ is well defined. Also, it is standard to prove that $J$ is a $C^{1}$ functional and

$$
J^{\prime}(u) v=\int_{\mathbb{R}}(-\Delta)^{1 / 4} u(-\Delta)^{1 / 4} v \mathrm{~d} x+\int_{\mathbb{R}} u v \mathrm{~d} x-\int_{\mathbb{R}} f(u) v \mathrm{~d} x, \quad \forall u, v \in H^{1 / 2}(\mathbb{R}) .
$$

Thus, the critical points of $J$ are precisely the solutions of (1.1), namely $u \in H^{1 / 2}(\mathbb{R})$ with

$$
\int_{\mathbb{R}}(-\Delta)^{1 / 4} u(-\Delta)^{1 / 4} v \mathrm{~d} x+\int_{\mathbb{R}} u v \mathrm{~d} x=\int_{\mathbb{R}} f(u) v \mathrm{~d} x, \quad \forall v \in H^{1 / 2}(\mathbb{R}),
$$

is a (weak) solution to (1.1). 
Lemma 2.2. Let $u \in H^{1 / 2}(\mathbb{R})$ and $\rho_{0}>0$ be such that $\|u\| \leq \rho_{0}$. Then

$$
\int_{\mathbb{R}}\left(e^{\alpha u^{2}}-1\right) \mathrm{d} x \leq \Lambda\left(\alpha, \rho_{0}\right), \quad \text { for every } 0<\alpha \rho_{0}^{2}<\omega ;
$$

Proof. Let $0<\alpha \rho_{0}^{2}<\omega$. Then, by Theorem A, we have

$$
\int_{\mathbb{R}}\left(e^{\alpha u^{2}}-1\right) \mathrm{d} x \leq \int_{\mathbb{R}}\left(e^{\alpha \rho_{0}^{2}\left(\frac{u}{\|u\|}\right)^{2}}-1\right) \mathrm{d} x \leq H_{\alpha \rho_{0}^{2}} \frac{\|u\|_{L^{2}}^{2}}{\|u\|^{2}} \leq H_{\alpha \rho_{0}^{2}}:=\Lambda\left(\alpha, \rho_{0}\right),
$$

since $\left\|(-\Delta)^{1 / 4} u\right\| u\left\|^{-1}\right\|_{L^{2}}^{2}<1$, see inequality (2.4).

Remark 2.3. From assumptions (f1)-(f2) and (AR) we see that, for all $s \in \mathbb{R} \backslash\{0\}$,

$$
\begin{aligned}
s^{2} f^{\prime}(s)-s f(s) & >0, \\
f^{\prime}(s) & >0, \\
\mathcal{H}(s):=s f(s)-2 F(s) & >0,
\end{aligned}
$$

$\mathcal{H}$ is even, and increasing on $\mathbb{R}^{+}$,

$$
\mathcal{H}(s)>\mathcal{H}(\lambda s), \quad \text { for all } \lambda \in(0,1) .
$$

Suppose that $u \neq 0$ is a critical point of $J$, that is, $J^{\prime}(u)=0$, then necessarily $u$ belongs to

$$
\mathcal{N}:=\left\{u \in H^{1 / 2}(\mathbb{R}) \backslash\{0\}: J^{\prime}(u) u=0\right\} .
$$

So $\mathcal{N}$ is a natural constraint for the problem of finding nontrivial critical points of $J$.

Lemma 2.4. Under assumptions (f1)-(f3) and (AR), $\mathcal{N}$ satisfies the following properties:

(a) $\mathcal{N}$ is a manifold and $\mathcal{N} \neq \emptyset$.

(b) For $u \in H^{1 / 2}(\mathbb{R}) \backslash\{0\}$ with $J^{\prime}(u) u<0$, there is a unique $\lambda(u) \in(0,1)$ with $\lambda u \in \mathcal{N}$.

(c) There exists $\rho>0$ such that $\|u\| \geq \rho$ for any $u \in \mathcal{N}$.

(d) If $u \in \mathcal{N}$ is a constrained critical point of $\left.J\right|_{\mathcal{N}}$, then $J^{\prime}(u)=0$ and $u$ solves (1.1).

(e) $m=\inf _{u \in \mathcal{N}} J(u)>0$.

Proof. Consider the $C^{1}$-functional $\Phi: H^{1 / 2}(\mathbb{R}) \backslash\{0\} \rightarrow \mathbb{R}$ defined by

$$
\Phi(u)=J^{\prime}(u) u=\|u\|^{2}-\int_{\mathbb{R}} f(u) u \mathrm{~d} x .
$$

Note that $\mathcal{N}=\Phi^{-1}(0)$ and $\Phi^{\prime}(u) u<0$, if $u \in \mathcal{N}$. Indeed, if $u \in \mathcal{N}$, then

$$
\Phi^{\prime}(u) u=\int_{\mathbb{R}}\left(f(u) u-f^{\prime}(u) u^{2}\right) \mathrm{d} x<0,
$$

where we have used (2.5). Then $c=0$ is regular value of $\Phi$ and consequently $\mathcal{N}=\Phi^{-1}(0)$ is a $C^{1}$-manifold, proving $(a)$. Now we prove $\mathcal{N} \neq \emptyset$ and that $(b)$ holds. Fix $u \in H^{1 / 2}(\mathbb{R}) \backslash\{0\}$ and consider the function $\Psi: \mathbb{R}^{+} \rightarrow \mathbb{R}$,

$$
\Psi(t)=\frac{t^{2}}{2}\|u\|^{2}-\int_{\mathbb{R}} F(t u) \mathrm{d} x .
$$

Then $\Psi^{\prime}(t)=0$ if and only if $t u \in \mathcal{N}$, in which case it holds

$$
\|u\|^{2}=\int_{\mathbb{R}} \frac{f(t u)}{t} u \mathrm{~d} x .
$$

In light of (2.5) the function on the right-hand side of $(2.10)$ is increasing. Whence, it follows that a critical point of $\Psi$, if it exists, it is unique. Now, there exist $\delta>0$ and $R>0$ such that

$$
\Psi(t)>0 \quad \text { if } t \in(0, \delta) \text { and } \Psi(t)<0 \quad \text { if } t \in(R, \infty) .
$$


In fact, by virtue of (f3), there exist $C, C^{\prime}>0$ such that

$$
\Psi(t)=\frac{t^{2}}{2}\|u\|^{2}-\int_{\mathbb{R}} F(t u) \mathrm{d} x \leq C t^{2}-C^{\prime} t^{q}<0,
$$

provided that $t>0$ is chosen large enough. Using (f1) and the fact that $f$ has $\alpha_{0}$-Trudinger-Moser critical growth at $+\infty$, for some $\alpha \in\left(\alpha_{0}, \omega\right)$ and for any $\varepsilon>0$, there exists $C_{\varepsilon}>0$ such that

$$
F(s) \leq \varepsilon\left[s^{2}+s^{4}\left(e^{\alpha s^{2}}-1\right)\right]+C_{\varepsilon} s^{4}, \quad s \in \mathbb{R} .
$$

Then, for any $u \in H^{1 / 2}(\mathbb{R}) \backslash\{0\}$,

$$
\Psi(t) \geq \frac{t^{2}}{2}\|u\|^{2}-\varepsilon t^{2}\|u\|_{L^{2}}^{2}-C_{\varepsilon} t^{4}\|u\|_{L^{4}}^{4}-\varepsilon t^{4} \int_{\mathbb{R}} u^{4}\left(e^{\alpha(t u)^{2}}-1\right) \mathrm{d} x .
$$

For $0<t<\tau<\left(\omega /\left(2 \alpha\|u\|^{2}\right)\right)^{1 / 2}$, by Lemma 2.2 and $(2.2)$, there is $C=C(\|u\|, \alpha)>0$ such that

$$
\int_{\mathbb{R}} u^{4}\left(e^{\alpha(t u)^{2}}-1\right) \leq\|u\|_{L^{8}}^{4}\left(\int_{\mathbb{R}} e^{2 \alpha \tau^{2} u^{2}}-1\right)^{1 / 2} \leq C .
$$

Then for some $B, B^{\prime}>0$, we have

$$
\Psi(t) \geq B t^{2}-B^{\prime} t^{4}>0, \quad \text { for } t>0 \text { small enough. }
$$

Thus, we conclude that there exists a unique maximum $t_{0}=t_{0}(u)>0$ such that $t_{0} u \in \mathcal{N}$, and consequently $\mathcal{N}$ is a nonempty set. Given $u \in H^{1 / 2}(\mathbb{R}) \backslash\{0\}$ with $J^{\prime}(u) u<0$, we have

$$
\Psi^{\prime}(1)=\|u\|^{2}-\int_{\mathbb{R}} f(u) u \mathrm{~d} x=J^{\prime}(u) u<0,
$$

which implies $t_{0}<1$. Let us prove (c). Let $\alpha \in\left(\alpha_{0}, \omega\right)$ and $\rho_{0}>0$ with $\alpha \rho_{0}^{2}<\omega$. By the growth conditions on $f$, there exists $r>1$ so close to 1 that $r \alpha \rho_{0}^{2}<\omega, \ell>2$ and $C>0$ with

$$
f(s) s \leq \frac{1}{4} s^{2}+C\left(e^{r \alpha s^{2}}-1\right)^{1 / r}|s|^{\ell}, \quad \text { for all } s \in \mathbb{R} .
$$

Let now $u \in \mathcal{N}$ with $\|u\| \leq \rho \leq \rho_{0}$. Then, by Lemma 2.2 and (2.2), we have for $u \in \mathcal{N}$

$$
\begin{aligned}
0 & =\Phi(u) \geq\|u\|^{2}-\frac{1}{4}\|u\|_{L^{2}}^{2}-C \int_{\mathbb{R}}\left(e^{r \alpha u^{2}}-1\right)^{1 / r}|u|^{\ell} \mathrm{d} x \\
& \geq \frac{3}{4}\|u\|^{2}-C\left(\int_{\mathbb{R}}\left(e^{r \alpha u^{2}}-1\right) \mathrm{d} x\right)^{1 / r}\left(\int_{\mathbb{R}}|u|^{r^{\prime} \ell} \mathrm{d} x\right)^{1 / r^{\prime}} \\
& \geq \frac{3}{4}\|u\|^{2}-C\|u\|^{\ell},
\end{aligned}
$$

which yields

$$
0<\hat{\rho}:=\left(\frac{3}{4 C}\right)^{1 /(\ell-2)} \leq\|u\| \leq \rho,
$$

a contradiction if $\rho<\min \left\{\hat{\rho}, \rho_{0}\right\}$. Then $u \in \mathcal{N}$ implies $\|u\| \geq \min \left\{\hat{\rho}, \rho_{0}\right\}$, proving (c). Concerning (d), if $u \in \mathcal{N}$ is a minimizer, then $J^{\prime}(u)=\lambda \Phi^{\prime}(u)$ for some $\lambda \in \mathbb{R}$. Testing with $u$ and recalling the previous conclusions yields $\lambda=0$, hence the assertion. Finally, assertion (e) follows by condition $(\mathbf{A R})$ and $(\mathrm{c})$, since $u \in \mathcal{N}$ implies $J(u) \geq(1 / 2-1 / \vartheta)\|u\|^{2} \geq(1 / 2-1 / \vartheta) \rho^{2}>0$.

Lemma 2.5. Let $\left(u_{n}\right) \subset \mathcal{N}$ be a minimizing sequence for $J$ on $\mathcal{N}$, that is,

$$
J^{\prime}\left(u_{n}\right) u_{n}=0 \quad \text { and } \quad J\left(u_{n}\right) \rightarrow m:=\inf _{u \in \mathcal{N}} J(u) \quad \text { as } n \rightarrow \infty,
$$

then the following facts hold

(a) $\left(u_{n}\right)$ is bounded in $H^{1 / 2}(\mathbb{R})$. Thus, up to a subsequence, $u_{n} \rightarrow u$ weakly in $H^{1 / 2}(\mathbb{R})$.

(b) $\lim \sup _{n}\left\|u_{n}\right\|<\rho_{0}$, for some $\rho_{0}>0$ sufficiently small. 
(c) $\left(u_{n}\right)$ does not converge strongly to zero in $L^{\sigma}(\mathbb{R})$, for some $\sigma>2$.

Proof. Let $\left(u_{n}\right) \subset H^{1 / 2}(\mathbb{R})$ satisfying (2.12). Using (AR) condition, we have for $\vartheta>2$,

$$
m+o(1)=J\left(u_{n}\right) \geq \frac{\left\|u_{n}\right\|^{2}}{2}-\frac{1}{\vartheta} \int_{\mathbb{R}} f\left(u_{n}\right) u_{n} \mathrm{~d} x=\left(\frac{1}{2}-\frac{1}{\vartheta}\right)\left\|u_{n}\right\|^{2},
$$

which implies (a). To prove (b) we use assumption (f3) and the fact that, by (2.2),

$$
\mathcal{S}_{q}:=\inf _{v \in H^{1 / 2}(\mathbb{R}) \backslash\{0\}} \mathcal{S}_{q}(v)>0, \quad \mathcal{S}_{q}(v)=\frac{\|v\|}{\|v\|_{L^{q}}} .
$$

Let $\left(u_{n}\right) \subset \mathcal{N}$ and $u \in \mathcal{N}$ satisfying (2.12). Then inequality (2.13) yields

$$
\limsup _{n}\left\|u_{n}\right\|^{2} \leq \frac{2 \vartheta}{\vartheta-2} m
$$

Notice that, for every $v \in H^{1 / 2}(\mathbb{R}) \backslash\{0\}$, arguing as for the proof of (b) of Lemma 2.4, one finds $t_{0}>0$ such that $t_{0} v \in \mathcal{N}$. Hence

$$
m \leq J\left(t_{0} v\right) \leq \max _{t \geq 0} J(t v)
$$

Now, using assumption (f3) and formula (2.14), for every $\psi \in H^{1 / 2}(\mathbb{R}) \backslash\{0\}$, we can estimate

$$
\begin{aligned}
m & \leq \max _{t \geq 0} J(t \psi) \leq \max _{t \geq 0}\left(\frac{t^{2}}{2}\|\psi\|^{2}-C_{q} t^{q}\|\psi\|_{L^{q}}^{q}\right) \\
& \leq \max _{t \geq 0}\left(\frac{\mathcal{S}_{q}(\psi)^{2}}{2} t^{2}\|\psi\|_{L^{q}}^{2}-C_{q} t^{q}\|\psi\|_{L^{q}}^{q}\right) \\
& =\left(\frac{1}{2}-\frac{1}{q}\right) \frac{\mathcal{S}_{q}(\psi)^{2 q /(q-2)}}{\left(q C_{q}\right)^{2 /(q-2)}},
\end{aligned}
$$

which together with (2.15) implies that

$$
\limsup _{n}\left\|u_{n}\right\|^{2} \leq \frac{2 \vartheta}{\vartheta-2}\left(\frac{1}{2}-\frac{1}{q}\right) \frac{\mathcal{S}_{q}(\psi)^{2 q /(q-2)}}{\left(q C_{q}\right)^{2 /(q-2)}} .
$$

Taking the infimum over $\psi \in H^{1 / 2}(\mathbb{R}) \backslash\{0\}$, we get

$$
\limsup _{n}\left\|u_{n}\right\|^{2} \leq \frac{\vartheta}{\vartheta-2} \frac{q-2}{q} \frac{\mathcal{S}_{q}^{2 q /(q-2)}}{\left(q C_{q}\right)^{2 /(q-2)}}<\rho_{0}^{2},
$$

provided $C_{q}$ is large enough, proving (b). Let us prove (c). By Lemma 2.4 (part (c)) we have

$$
\left\|u_{n}\right\|^{2}=\int_{\mathbb{R}} f\left(u_{n}\right) u_{n} \mathrm{~d} x \geq \rho^{2}>0 .
$$

In view of assertion (b) the norm $\left\|u_{n}\right\|$ is small (precisely, we can assumed that $r \alpha\left\|u_{n}\right\|^{2}<r \alpha \rho_{0}^{2}<\omega$ for $r$ very close to 1$)$. Arguing as in the proof of $(2.11)$, we can find $\varepsilon \in(0,1)$ and $C>0$ such that

$$
\begin{aligned}
\left\|u_{n}\right\|^{2}=\int_{\mathbb{R}} f\left(u_{n}\right) u_{n} \mathrm{~d} x & \leq \varepsilon\left\|u_{n}\right\|_{L^{2}}^{2}+C \int_{\mathbb{R}}\left(e^{r \alpha u_{n}^{2}}-1\right)^{1 / r}\left|u_{n}\right|^{\ell} \mathrm{d} x \\
& \leq \varepsilon\left\|u_{n}\right\|^{2}+C\left(\int_{\mathbb{R}}\left(e^{r \alpha u_{n}^{2}}-1\right) \mathrm{d} x\right)^{1 / r}\left\|u_{n}\right\|_{L^{r^{\prime} \ell}}^{\ell} \\
& \leq \varepsilon\left\|u_{n}\right\|^{2}+C\left\|u_{n}\right\|_{L^{r^{\prime} \ell}}^{\ell},
\end{aligned}
$$

which implies

$$
0<(1-\varepsilon) \rho^{2} \leq(1-\varepsilon)\left\|u_{n}\right\|^{2} \leq C\left\|u_{n}\right\|_{L^{r^{\prime} \ell}}^{\ell}
$$


and, consequently, $\left(u_{n}\right)$ cannot vanish in $L^{r^{\prime} \ell}(\mathbb{R})$, as $n \rightarrow \infty$. This concludes the proof. Next, we formulate a Brezis-Lieb type lemma in our framework.

Lemma 2.6. Let $\left(u_{n}\right) \subset H^{1 / 2}(\mathbb{R})$ be a sequence such that $u_{n} \rightarrow u$ weakly in $H^{1 / 2}(\mathbb{R})$ and $\left\|u_{n}\right\|<\rho_{0}$ with $\rho_{0}>0$ small. Then, as $n \rightarrow \infty$, we have

$$
\begin{aligned}
\int_{\mathbb{R}} f\left(u_{n}\right) u_{n} \mathrm{~d} x & =\int_{\mathbb{R}} f\left(u_{n}-u\right)\left(u_{n}-u\right) \mathrm{d} x+\int_{\mathbb{R}} f(u) u \mathrm{~d} x+o(1), \\
\int_{\mathbb{R}} F\left(u_{n}\right) \mathrm{d} x & =\int_{\mathbb{R}} F\left(u_{n}-u\right) \mathrm{d} x+\int_{\mathbb{R}} F(u) \mathrm{d} x+o(1) .
\end{aligned}
$$

Proof. We shall apply [8, Lemma 3 and Theorem 2$]$. Since $f$ is convex on $\mathbb{R}^{+}$and by the properties collected in Remark 2.3, we have that the functions $F(s)$ and $G(s):=f(s) s$ are convex on $\mathbb{R}$ with $F(0)=G(0)=0$. We let $\alpha \in\left(\alpha_{0}, \omega\right)$ and $\rho_{0} \in(0,1 / 2)$ with $\alpha \rho_{0}^{2}<\omega$. Thus, by Lemma 2.2, we have

$$
\sup _{n \in \mathbb{N}} \int_{\mathbb{R}}\left(e^{\alpha u_{n}^{2}}-1\right) \mathrm{d} x<\infty .
$$

Choose $k \in\left(1, \frac{1-\rho_{0}}{\rho_{0}}\right)$ and let $\varepsilon>0$ with $\varepsilon<1 / k$. Then, in light of [8, Lemma 3], the functions

$$
\phi_{\varepsilon}(s):=j(k s)-k j(s) \geq 0 \quad \psi_{\varepsilon}(s):=\left|j\left(C_{\varepsilon} s\right)\right|+\left|j\left(-C_{\varepsilon} s\right)\right|, \quad s \in \mathbb{R}, \quad C_{\varepsilon}=\frac{1}{\varepsilon(k-1)},
$$

satisfy the inequality

$$
|j(a+b)-j(a)| \leq \varepsilon \phi_{\varepsilon}(a)+\psi_{\varepsilon}(b), \quad \forall a, b \in \mathbb{R},
$$

and, if $v_{n}:=u_{n}-u$ and $u_{n}$ satisfying (2.17), we claim that

(i) $v_{n} \rightarrow 0$ almost everywhere;

(ii) $j(u) \in L^{1}(\mathbb{R})$;

(iii) $\int_{\mathbb{R}} \phi_{\varepsilon}\left(v_{n}\right) d x \leq C$ for some constant $C$ independent of $n \geq 1$;

(iv) $\int_{\mathbb{R}} \psi_{\varepsilon}(u) d x<\infty$, for all $\varepsilon>0$ small.

Assuming this claim, then, by [8, Theorem 2], it holds

$$
\lim _{n} \int_{\mathbb{R}}\left|j\left(u_{n}\right)-j\left(v_{n}\right)-j(u)\right| d x=0
$$

with $j=F$ and with $j=G$. Next we are going to prove the claim. Item (i) follows by the week convergence of $\left(u_{n}\right)$. To prove (ii) it is enough to use Proposition 2.1 (see the growth conditions below). To check (iii) for $j=F$ and $j=G$, we find $\alpha \in\left(\alpha_{0}, \omega\right), D>0$ and $q>2$ such that

$$
\begin{aligned}
F(s) & \leq\left(s^{2}+e^{\alpha s^{2}}-1\right)+D|s|^{q}, \quad \text { for all } s \in \mathbb{R}, \\
G(s) & \leq\left(s^{2}+e^{\alpha s^{2}}-1\right)+D|s|^{q}, \quad \text { for all } s \in \mathbb{R}, \\
|f(s)| & \leq\left(s+e^{\alpha s^{2}}-1\right)+D|s|^{q-1}, \quad \text { for all } s \in \mathbb{R}, \\
\left|f^{\prime}(s) s\right| & \leq\left(s+e^{\alpha s^{2}}-1\right)+D|s|^{q-1}, \quad \text { for all } s \in \mathbb{R} .
\end{aligned}
$$

We claim that $\phi_{\varepsilon}\left(v_{n}\right)$ verifies (iii). First let us consider the case $j=F$, that is,

$$
\phi_{\varepsilon}\left(v_{n}\right)=F\left(k v_{n}\right)-k F\left(v_{n}\right) .
$$

In fact, by the Mean Value Theorem, there exists $\vartheta \in(0,1)$ with $w_{n}=v_{n}(k(1-\vartheta)+\vartheta)$ such that

$$
\begin{aligned}
\phi_{\varepsilon}\left(v_{n}\right) & =F\left(k v_{n}\right)-F\left(v_{n}\right)+F\left(v_{n}\right)-k F\left(v_{n}\right) \\
& =f\left(w_{n}\right) v_{n}(k-1)+(1-k) F\left(v_{n}\right) \leq f\left(w_{n}\right) v_{n}(k-1),
\end{aligned}
$$


since $k>1$ and $F \geq 0$. Analogously, for $j=G$, we have

$$
\begin{aligned}
\phi_{\varepsilon}\left(v_{n}\right) & =G\left(k v_{n}\right)-G\left(v_{n}\right)+G\left(v_{n}\right)-k G\left(v_{n}\right) \\
& =f^{\prime}\left(w_{n}\right) w_{n} v_{n}(k-1)+f\left(w_{n}\right) v_{n}(k-1)+(1-k) f\left(v_{n}\right) v_{n} \\
& \leq f^{\prime}\left(w_{n}\right) w_{n} v_{n}(k-1)+f\left(w_{n}\right) v_{n}(k-1),
\end{aligned}
$$

since $k>1$ and $f(s) s \geq 0$ for all $s \in \mathbb{R}$. Thus, to prove (iii) for $F$ and $G$, it is sufficient to see that

$$
\sup _{n \in \mathbb{N}} \int_{\mathbb{R}} f\left(w_{n}\right) v_{n} \mathrm{~d} x<\infty, \quad \sup _{n \in \mathbb{N}} \int_{\mathbb{R}} f^{\prime}\left(w_{n}\right) w_{n} v_{n} \mathrm{~d} x<\infty .
$$

We know that

$$
\left\|u_{n}\right\|^{2}=\left\|v_{n}\right\|^{2}+\|u\|^{2}+o(1), \text { as } n \rightarrow \infty,
$$

so that $\lim \sup _{n}\left\|v_{n}\right\| \leq \rho_{0}$. In turn, by the choice of $k$, we also have

$$
\limsup _{n}\left\|w_{n}\right\|=\left\|v_{n}\right\|(k(1-\vartheta)+\vartheta) \leq \rho_{0}(k(1-\vartheta)+\vartheta) \leq \rho_{0}(k+1)<1 .
$$

Since $\alpha_{0}<\alpha<\omega$, we can find $m>1$ very close to 1 such that $m \alpha<\omega$. Then, by (2.22), we get

$$
\begin{aligned}
\int_{\mathbb{R}} f\left(w_{n}\right) v_{n} & \leq \int_{\mathbb{R}}\left|w_{n} \| v_{n}\right| \mathrm{d} x+\int_{\mathbb{R}}\left(e^{\alpha w_{n}^{2}}-1\right)\left|v_{n}\right| \mathrm{d} x+D \int_{\mathbb{R}}\left|w_{n}\right|^{q-1}\left|v_{n}\right| \mathrm{d} x \\
& \leq\left\|w_{n}\right\|_{L^{2}}\left\|v_{n}\right\|_{L^{2}}+D\left\|w_{n}\right\|_{L^{q}}^{q-1}\left\|v_{n}\right\|_{L^{q}}+\left(\int_{\mathbb{R}}\left(e^{m \alpha w_{n}^{2}}-1\right) \mathrm{d} x\right)^{1 / m}\left\|v_{n}\right\|_{L^{m^{\prime}}} \\
& \leq C\left\|w_{n}\right\|\left\|v_{n}\right\|+C\left\|w_{n}\right\|^{q-1}\left\|v_{n}\right\|+C\left(\int_{\mathbb{R}}\left(e^{m \alpha w_{n}^{2}}-1\right) \mathrm{d} x\right)^{1 / m}\left\|v_{n}\right\| \\
& \leq C+C\left(\int_{\mathbb{R}}\left(e^{m \alpha w_{n}^{2}}-1\right) \mathrm{d} x\right)^{1 / m} \leq C .
\end{aligned}
$$

The last integral is bounded via Lemma 2.2 , since $\left\|w_{n}\right\| \leq 1$ and $m \alpha<\omega$. The second term in (2.24) can be treated in a similar fashion, using the growth condition (2.23) in place of (2.22). We claim that $\psi_{\varepsilon}$ verifies (iv) for both $F$ and $G$. It suffices to prove

$$
\int_{\mathbb{R}} F\left(C_{\varepsilon} u\right) \mathrm{d} x<\infty, \text { for all } \varepsilon>0 .
$$

By (2.20) this occurs since by Proposition 2.1, we have

$$
\int_{\mathbb{R}}\left(e^{\alpha C_{\varepsilon}^{2} u^{2}}-1\right) \mathrm{d} x<\infty .
$$

Analogous proof holds for $G$ via (2.21). We can finally apply [8, Theorem 2] yielding (2.19). Thus

$$
\int_{\mathbb{R}} j\left(u_{n}\right) \mathrm{d} x=\int_{\mathbb{R}} j\left(v_{n}\right) \mathrm{d} x+\int_{\mathbb{R}} j(u) \mathrm{d} x+o(1),
$$

for $j=F$ and $j=G$. This concludes the proof.

The previous Lemma 2.6 yields the following useful technical results.

Lemma 2.7. Let $\left(u_{n}\right) \subset H^{1 / 2}(\mathbb{R})$ be as in Lemma 2.5 then for $v_{n}=u_{n}-u$ we have

$$
J^{\prime}(u) u+\liminf _{n} J^{\prime}\left(v_{n}\right) v_{n}=0,
$$

so that either $J^{\prime}(u) u \leq 0$ or $\liminf _{n} J^{\prime}\left(v_{n}\right) v_{n}<0$. 
Proof. Recalling that $v_{n}=u_{n}-u$, we get $\left\|u_{n}\right\|^{2}=\left\|v_{n}\right\|^{2}+\|u\|^{2}+o(1)$. Then by Lemma 2.6,

$$
\int_{\mathbb{R}} f\left(u_{n}\right) u_{n} \mathrm{~d} x=\int_{\mathbb{R}} f\left(v_{n}\right) v_{n} \mathrm{~d} x+\int_{\mathbb{R}} f(u) u \mathrm{~d} x+o(1) .
$$

Since $u_{n} \in \mathcal{N}$, by using the above equality, the assertion follows.

Lemma 2.8. Let $\left(u_{n}\right) \subset \mathcal{N}$ be a minimizing sequence for $J$ on $\mathcal{N}$, such that $u_{n} \rightarrow u$ weakly in $H^{1 / 2}(\mathbb{R})$ as $n \rightarrow \infty$. If $u \in \mathcal{N}$, then $J(u)=m$.

Proof. Let $\left(u_{n}\right) \subset \mathcal{N}$ and $u \in \mathcal{N}$ as above, thus

$$
m+o(1)=J\left(u_{n}\right)-\frac{1}{2} J^{\prime}\left(u_{n}\right) u_{n}=\frac{1}{2} \int_{\mathbb{R}} \mathcal{H}\left(u_{n}\right) \mathrm{d} x
$$

which together with Fatou's lemma (recall that (2.7) holds) implies

$$
m=\frac{1}{2} \liminf _{n} \int_{\mathbb{R}} \mathcal{H}\left(u_{n}\right) \mathrm{d} x \geq \frac{1}{2} \liminf _{n} \int_{\mathbb{R}} \mathcal{H}(u) \mathrm{d} x=J(u)-\frac{1}{2} J^{\prime}(u) u=J(u),
$$

which yields the conclusion.

\section{Proof of Theorem 1.1 Concluded}

Let $\left(u_{n}\right) \subset \mathcal{N}$ be a minimizing sequence for $J$ on $\mathcal{N}$. From Lemma $2.5\left(\right.$ a), $\left(u_{n}\right)$ is bounded in $H^{1 / 2}(\mathbb{R})$. Thus, up to a subsequence, we have $u_{n} \rightarrow u$ weakly in $H^{1 / 2}(\mathbb{R})$.

Assertion 3.1. There exist a sequence $\left(y_{n}\right) \subset \mathbb{R}$ and constants $\gamma, R>0$ such that

$$
\liminf _{n} \int_{y_{n}-R}^{y_{n}+R}\left|u_{n}\right|^{2} \mathrm{~d} x \geq \gamma>0
$$

If not, for any $R>0$,

$$
\liminf _{n} \sup _{y \in \mathbb{R}} \int_{y-R}^{y+R}\left|u_{n}\right|^{2} \mathrm{~d} x=0 .
$$

Using a standard concentration-compactness principle due to P.L. Lions (it is easy to see that the argument remains valid for the case studied here) we can conclude that $u_{n} \rightarrow 0$ in $L^{q}(\mathbb{R})$ for any $q>2$, which is a contradiction with Lemma 2.5 (c). Define $\bar{u}_{n}(x)=u_{n}\left(x+y_{n}\right)$. Then $J\left(u_{n}\right)=J\left(\bar{u}_{n}\right)$ and without of loss generality we can assume $y_{n}=0$ for any $n$. Notice that $\left(\bar{u}_{n}\right)$ is also a minimizing sequence for $J$ on $\mathcal{N}$, which it is bounded and satisfies

$$
\liminf _{n} \int_{-R}^{R}\left|\bar{u}_{n}\right|^{2} \mathrm{~d} x \geq \gamma, \quad \text { for some } \quad \gamma>0,
$$

and $\bar{u}_{n} \rightarrow \bar{u}$ weakly in $H^{1 / 2}(\mathbb{R})$, then $\bar{u} \neq 0(u \neq 0)$.

Assertion 3.2. $J^{\prime}(u) u=0$.

If Assertion 3.2 holds, then combining (d) of Lemma 2.4 and Lemma 2.8 we have the result.

We shall now prove Assertion 3.2. Suppose by contradiction that $J^{\prime}(u) u \neq 0$.

- If $J^{\prime}(u) u<0$, by Lemma 2.4 (b), there exists $0<\lambda<1$ such that $\lambda u \in \mathcal{N}$. Thus

$$
\lambda\|u\|^{2}=\int_{\mathbb{R}} f(\lambda u) u \mathrm{~d} x
$$

Using (2.7) in combination with Fatou's Lemma, we obtain

$$
m=\liminf _{n} \frac{1}{2} \int_{\mathbb{R}} \mathcal{H}\left(u_{n}\right) \mathrm{d} x \geq \frac{1}{2} \int_{\mathbb{R}} \mathcal{H}(u) \mathrm{d} x>\frac{1}{2} \int_{\mathbb{R}} \mathcal{H}(\lambda u) \mathrm{d} x=J(\lambda u)-\frac{1}{2} J^{\prime}(\lambda u) \lambda u=J(\lambda u),
$$


which implies that $J(\lambda u)<m$ and, hence, a contradiction. Here we have used (2.9).

- If $J^{\prime}(u) u>0$, by Lemma 2.7, we get $\liminf { }_{n} J^{\prime}\left(v_{n}\right) v_{n}<0$. Taking a subsequence, we have $J^{\prime}\left(v_{n}\right) v_{n}<0$, for $n$ large. By Lemma $2.4(\mathrm{~b})$, there exists $\lambda_{n} \in(0,1)$ such that $\lambda_{n} v_{n} \in \mathcal{N}$.

Assertion 3.3. $\lim \sup _{n} \lambda_{n}<1$.

If $\lim \sup _{n} \lambda_{n}=1$, up to a sub-sequence, we can assume that $\lambda_{n} \rightarrow 1$, then

$$
J^{\prime}\left(v_{n}\right) v_{n}=J^{\prime}\left(\lambda_{n} v_{n}\right) \lambda_{n} v_{n}+o(1) .
$$

This follows provided that

$$
\int_{\mathbb{R}} f\left(v_{n}\right) v_{n} \mathrm{~d} x=\int_{\mathbb{R}} f\left(\lambda_{n} v_{n}\right) \lambda_{n} v_{n} \mathrm{~d} x+o(1) .
$$

In fact, notice that if $\eta_{n}:=v_{n}+\tau v_{n}\left(\lambda_{n}-1\right)$ for some $\tau \in(0,1)$, it follows

$$
f\left(v_{n}\right) v_{n}-f\left(\lambda_{n} v_{n}\right) \lambda_{n} v_{n}=\left(f^{\prime}\left(\eta_{n}\right) \eta_{n}+f\left(\eta_{n}\right)\right) v_{n}\left(1-\lambda_{n}\right) .
$$

Since $\left\|\eta_{n}\right\|=\left\|v_{n}+\tau v_{n}\left(\lambda_{n}-1\right)\right\| \leq \lambda_{n}\left\|v_{n}\right\| \leq \rho_{0}$, it follows by arguing as for the justification of formula (2.24), that

$$
\sup _{n \in \mathbb{N}} \int_{\mathbb{R}}\left|f^{\prime}\left(\eta_{n}\right) \eta_{n}+f\left(\eta_{n}\right)\right|\left|v_{n}\right| \mathrm{d} x<\infty,
$$

so that (3.2) follows, since $\lambda_{n} \rightarrow 1$. Since $\lambda_{n} v_{n} \in \mathcal{N}$ we have $J^{\prime}\left(\lambda_{n} v_{n}\right) \lambda_{n} v_{n}=0$ which implies that

$$
J^{\prime}\left(v_{n}\right) v_{n}=o(1),
$$

which is a contradiction with $\lim _{n} J^{\prime}\left(v_{n}\right) v_{n}<0$. Thus, up to subsequence, we may assume that $\lambda_{n} \rightarrow \lambda_{0} \in(0,1)$. Arguing as before, from (2.9) we infer

$$
m+o(1)=\frac{1}{2} \int_{\mathbb{R}} \mathcal{H}\left(u_{n}\right) \mathrm{d} x \geq \frac{1}{2} \int_{\mathbb{R}} \mathcal{H}\left(\lambda_{n} u_{n}\right) \mathrm{d} x,
$$

since $\mathcal{H}\left(u_{n}\right) \geq \mathcal{H}\left(\lambda_{n} u_{n}\right)$. By means of Lemma 2.6 applied to $w_{n}=\lambda_{n} u_{n}$ (whose norm is small, being smaller than the norm of $u_{n}$ ) and $w=\lambda_{0} u$ we have in turn

$$
\int_{\mathbb{R}} \mathcal{H}\left(\lambda_{n} u_{n}\right) \mathrm{d} x=\int_{\mathbb{R}} \mathcal{H}\left(\lambda_{n} u_{n}-\lambda_{0} u\right) \mathrm{d} x+\int_{\mathbb{R}} \mathcal{H}\left(\lambda_{0} u\right) \mathrm{d} x+o(1) .
$$

Furthermore, we have

$$
\int_{\mathbb{R}} \mathcal{H}\left(\lambda_{n} u_{n}-\lambda_{0} u\right) \mathrm{d} x=\int_{\mathbb{R}} \mathcal{H}\left(\lambda_{n} v_{n}\right) \mathrm{d} x+o(1) .
$$

In fact, notice that $\lambda_{n} u_{n}-\lambda_{0} u=\lambda_{n} v_{n}+\gamma_{n} u$, where $\gamma_{n}:=\lambda_{n}-\lambda_{0} \rightarrow 0$ as $n \rightarrow \infty$. We have

$$
\mathcal{H}\left(\lambda_{n} u_{n}-\lambda_{0} u\right)-\mathcal{H}\left(\lambda_{n} v_{n}\right)=\mathcal{H}^{\prime}\left(\hat{\eta}_{n}\right) u \gamma_{n}, \quad \hat{\eta}_{n}:=\tau u \gamma_{n}+\lambda_{n} v_{n}
$$

for $\tau \in(0,1)$ and $\left\|\hat{\eta}_{n}\right\|=\left\|\tau u \gamma_{n}+\lambda_{n} v_{n}\right\| \leq \gamma_{n}\|u\|+\lambda_{n}\left\|v_{n}\right\| \leq \rho_{0}$ for $n$ large. Then, arguing as for the justification of $(2.24)$, we get

$$
\sup _{n \in \mathbb{N}} \int_{\mathbb{R}}\left|\mathcal{H}^{\prime}\left(\hat{\eta}_{n}\right)\right||u| \mathrm{d} x \leq \sup _{n \in \mathbb{N}} \int_{\mathbb{R}}\left|f^{\prime}\left(\hat{\eta}_{n}\right) \hat{\eta}_{n}+f\left(\hat{\eta}_{n}\right)\right|\left|v_{n}\right| \mathrm{d} x<\infty
$$

which yields (3.3) since $\gamma_{n} \rightarrow 0$ as $n \rightarrow \infty$. Therefore, we obtain

$$
\begin{aligned}
m+o(1) & \geq \frac{1}{2} \int_{\mathbb{R}} \mathcal{H}\left(\lambda_{n} v_{n}\right) \mathrm{d} x+\frac{1}{2} \int_{\mathbb{R}} \mathcal{H}\left(\lambda_{0} u\right) \mathrm{d} x \\
& =J\left(\lambda_{n} v_{n}\right)-\frac{1}{2} J^{\prime}\left(\lambda_{n} v_{n}\right) \lambda v_{n}+\frac{1}{2} \int_{\mathbb{R}} \mathcal{H}\left(\lambda_{0} u\right) \mathrm{d} x=J\left(\lambda_{n} v_{n}\right)+\frac{1}{2} \int_{\mathbb{R}} \mathcal{H}\left(\lambda_{0} u\right) \mathrm{d} x .
\end{aligned}
$$

Since $u \neq 0$, we have $\int_{\mathbb{R}} \mathcal{H}\left(\lambda_{0} u\right) \mathrm{d} x>0$. Then $J\left(\lambda_{n} v_{n}\right)<m$ for large $n$, a contradiction. 


\section{REFERENCES}

[1] Adimurthi, S.L. Yadava, Multiplicity results for semilinear elliptic equations in a bounded domain of $\mathbb{R}^{2}$ involving critical exponent, Ann. Sc. Norm. Super. Pisa Cl. Sci. (1990), 481-504. 2

[2] C. Alves, J. M. do Ó, O. Miyagaki, On nonlinear perturbations of a periodic elliptic problem in $\mathbb{R}^{2}$ involving critical growth, Nonlinear Anal. 56 (2004), 781-791. 2

[3] C. Alves, M. Souto, M. Montenegro, Existence of a ground state solution for a nonlinear scalar field equation with critical growth, Calc. Var. Partial Differential Equations 43 (2012), 537-554. 2

[4] A. Ambrosetti, Z.-Q. Wang, Positive solutions to a class of quasilinear elliptic equations on $\mathbb{R}$, Discrete Contin. Dyn. Syst. 9 (2003), 55-68. 2

[5] B. Barrios, E. Colorado, A. de Pablo, U. Sánchez, On some critical problems for the fractional Laplacian operator, J. Differential Equations 252 (2012), 613-6162. 3

[6] H. Berestycki, P.-L. Lions, Nonlinear scalar field equations, I-existence of a ground state. Arch. Rat. Mech. Anal. 82 (1983), 313-346. 2, 3

[7] H. Berestycki, T. Gallouet, O. Kavian, Equations de Champs scalaires euclidiens non lineaires dans le plan, C. R. Acad. Sci. Paris Ser. I Math. 297 (1984), 307-310. 2

[8] H. Brezis, E. Lieb, A relation between pointwise convergence of functions and convergence of functionals, Proc. Amer. Math. Soc. 88 (1983), 486-490. 8, 9

[9] X. Cabré, J. G. Tan, Positive solutions of nonlinear problems involving the square root of the Laplacian, Adv. Math. 224 (2010), 2052-2093. 3

[10] X. Chang, Z-Q. Wang, Ground state of scalar field equations involving a fractional laplacian with general nonlinearity, Nonlinearity 26 (2013), 479-494. 2, 3

[11] D. Cao, Nontrivial solution of semilinear elliptic equation with critical exponent in $\mathbb{R}^{2}$, Comm. Partial Differential Equations 17(1992), 407-435. 2

[12] V. Coti Zelati, P. Rabinowitz, Homoclinic type solutions for a semilinear elliptic PDE on $\mathbb{R}^{N}$. Comm. Pure Appl. Math. 45 (1992), 1217-1269. 2

[13] D. G. de Figueiredo, J. M. do Ó, B. Ruf, Elliptic Equations and Systems with critical Trudinger-Moser nonlinearities, Discrete Contin. Dyn. Syst. 30 (2011), 455-476. 2

[14] D. G. de Figueiredo, O. H. Miyagaki, B. Ruf, Elliptic equations in $\mathbb{R}^{2}$ with nonlinearities in the critical growth range, Calc. Var. Partial Differential Equations 3 (1995), 139-153. 2

[15] D. G. De Figueiredo, J. M. do Ó, B. Ruf, On an inequality by N. Trudinger and J. Moser and related elliptic equations, Comm. Pure Appl. Math. 55 (2002), 135-152. 2

[16] E. Di Nezza, G. Palatucci, E. Valdinoci, Hitchhiker's guide to the fractional Sobolev spaces, Bull. Sci. Math. 136 (2012), 521-573. 3

[17] J. M. do Ó, E. Medeiros, U. Severo, A nonhomogeneous elliptic problem involving critical growth in dimension two, J. Math. Anal. Appl. 345 (2008), 286-304. 2

[18] J. M. do Ó, O. H. Miyagaki, M. Squassina, Critical and subcritical fractional problems with vanishing potentials, preprint 3

[19] J. M. do Ó, O. H. Miyagaki, M. Squassina, Nonautonomous fractional problems with exponential growth, NoDEA Nonlinear Differential Equations Applications, to appear. 2, 3

[20] P. Felmer, A. Quaas, J. Tan Positive solutions of nonlinear Schrödinger equation with the fractional Laplacian, Proc. Roy. Soc. Edinburgh A 142 (2012), 1237-1262. 3

[21] R. Frank, E. Lenzmann, Uniqueness of non-linear ground states for fractional laplacians in $\mathbb{R}$, Acta Math. 210 (2013), 261-318. 2, 3

[22] T. Jin, Y. Li, J. Xiong, On a fractional Nirenberg problem, part I: blow up analysis and compactness of solutions, J. Eur. Math. Soc. 16 (2014), 1111-1171. 3

[23] A. Iannizzotto, M. Squassina, 1/2-laplacian problems with exponential nonlinearity, J. Math. Anal. Appl. 414 (2014), 372-385. 2, 3

[24] M.A. Krasnosel'skiū, J.B. Rutickiǔ, Convex functions and Orlicz spaces, Noordhoff, Groningen, Holland, 1961. 4

[25] N. Lam, G. Lu, Elliptic equations and systems with subcritical and critical exponential growth without the Ambrosetti-Rabinowitz condition, J. Geom. Anal. 24 (2014), 118-143. 2

[26] E. H. Lieb, M. Loss, Analysis, AMS, 2001. 3

[27] J. Moser, A sharp form of an inequality by N. Trudinger, Indiana Univ. Math. J. 20 (1970), 1077-1092. 2

[28] T. Ozawa, On critical cases of Sobolev's inequalities, J. Funct. Anal. 127 (1995), 259-269. 1, 3

[29] B. Ruf, F. Sani, Ground states for elliptic equations in $\mathbb{R}^{2}$ with exponential critical growth. Geometric properties for parabolic and elliptic PDE's, 251-267, Springer INdAM 2, Springer, Milan, 2013. 2 
[30] X. Shang, J. Zhang, Y. Yang, On fractional Schödinger equation in $\mathbb{R}^{N}$ with critical growth, J. Math. Phys. 54 (2013), 121502-19 pages. 3

[31] S. Secchi, Ground state solutions for nonlinear fractional Schrödinger equations in $\mathbb{R}^{N}$, J. Math. Phys. 54 (2013), 031501-17 pages. 3

[32] W. Strauss, Existence of solitary waves in higher dimensions,. Comm. Math. Phys. 55 (2) (1977), 149-162. 2

[33] N. Trudinger, On imbeddings into Orlicz spaces and some applications, J. Math. Mech. 17 (1967), 473-483. 2

[34] M. Willem,Minimax Theorems, Progress in Nonlinear Differential Equations and their Applications, 24, Birkhäuser 1996. 2

(J.M. do Ó) Department of Mathematics, Federal University of Paraíba 58051-900, Jỗo PessoA-PB, BrazIL

E-mail address: jmbo@pq.cnpq.br

(O. Miyagaki) Department of Mathematics, Federal University of Juiz de Fora 36036-330 Juiz de Fora, Minas Gerais, Brazil

E-mail address: olimpio@ufv.br

(M. Squassina) Dipartimento di Informatica Università degli Studi di Verona, Cá Vignal 2, Strada Le Grazie 15, I-37134 Verona, Italy

E-mail address: marco.squassina@univr.it 